\title{
A beam search-based algorithm and evaluation of scheduling approaches for flexible manufacturing systems
}

\author{
IHSAN SABUNCUOGLU ${ }^{1}$ and SULEYMAN KARABUK ${ }^{2 *}$ \\ ${ }^{1}$ Department of Industrial Engineering, Bilkent University, Ankara 06533, Turkey \\ ${ }^{2}$ Department of Industrial Engineering, Lehigh University, Bethlehem, PA 18015, USA
}

Received September 1994 and accepted November 1997

\begin{abstract}
This paper presents a new algorithm for the flexible manufacturing system (FMS) scheduling problem. The proposed algorithm is a heuristic based on filtered beam search. It considers finite buffer capacity, routing and sequence flexibilities and generates machine and automated guided vehicle (AGV) schedules for a given scheduling period. A new deadlock resolution mechanism is also developed as an integral part of the proposed algorithm. The performance of the algorithm is compared with several machine and AGV dispatching rules using mean flow time, mean tardiness and makespan criteria. It is also used to examine the effects of scheduling factors (i.e., machine and AGV load levels, routing and sequence flexibilities, etc.) on the system performance. The results indicate that the proposed scheduling algorithm yields considerable improvements in system performance over dispatching rules under a wide variety of experimental conditions.
\end{abstract}

\section{Introduction}

As described by Rachamadugu and Stecke [1], flexible manufacturing system (FMS) is a general term used to refer to various types of automated manufacturing systems. In this paper, a new scheduling algorithm is proposed for a random FMS. The random FMS is a nondedicated flexible manufacturing system that can process a wide variety of different parts with low to medium demand volume. These systems are similar to conventional job shops. The differences are due to the high level of automation and considerations of additional resources as well as various types of flexibilities in a dynamic FMS environment.

The problem can be defined as detailed scheduling of the machines, automated guided vehicles (AGVs), and other supporting equipment for given shop conditions and a set of parts with known processing requirements. Scheduling decisions include: (1) determining job release times; (2) starting and completion times of operations; and (3) contingency plans in case of unexpected interruptions such as machine breakdowns. In general, the scheduling problem defined above is more difficult than the job shop problem. Even the two machine case is NPhard [2]. Over the last decade, this problem has been

\footnotetext{
*This research was done while S. Karabuk was at Bilkent University.
}

extensively studied with Basnet and Mize [3] and Rachamadugu and Stecke [1] currently providing the most up-to-date and comprehensive reviews in this area. It is evident from their remarks that most scheduling methods proposed for FMS scheduling problems consider only a few system resources and a limited number of factors.

In this paper, we propose an algorithm which considers most of the scheduling factors listed in the literature (i.e., machines, material handling, finite buffer capacity, routing and sequence flexibilities). Specifically, it utilizes system and job related information to generate machine and AGV schedules for an entire scheduling horizon as well as various lengths of scheduling periods (or time windows). The proposed algorithm is also used as a tool to examine interactions between factors and their effects on the system performance. Moreover, it is compared to several on-line scheduling rules using makespan, flow time and tardiness related criteria under different experimental conditions. Hence, this study also provides a detailed comparison between two well-known scheduling approaches (off-line and on-line methods).

The rest of the paper is organized as follows. The next section presents a literature review. In Section 3 the structure and characteristics of the proposed algorithm are described in detail. This is followed by systems considerations and experimental conditions in Section 4. The results of our experiments are presented in Section 5. The paper ends with concluding remarks and suggestions for further research in Section 6. 


\section{Literature review}

One of the earliest studies on the FMS scheduling problem is the work of Nof et al. [4] who demonstrated the importance of scheduling decisions for system performance. A considerable amount of literature has been produced since this initial paper. Most papers consider machines as the primary resource and use optimization approaches to solve FMS scheduling problems as for example, in the papers of Yamamoto and Nof [5] and Chang et al. [6]. The full complexity of the FMS problem (i.e., consideration of a material handling system, limited buffer capacities, routing and sequence flexibilities) has only been recently studied. For example, Han and McGinnis [7] have developed a real time algorithm in which material handling transporters are considered. Schriber and Stecke [8] have shown how the additional consideration of the material handling system and limited buffers degrades the system performance. Sabuncuoglu and Hommertzheim $[9,10]$ have highlighted the importance of material handling and they compared several AGV dispatching rules. They have also shown how the buffer capacity can affect the performance of the system.

Flexibility, which is a distinguishing feature of FMSs, has received an extensive amount of attention. Routing flexibility (i.e., alternative machines and processing routes) have been considered by Wilhelm and Shin [11], Chen and Chung [12], and Khoshnevis and Chen [13]. These studies have indicated that dynamic routing (i.e., a path determined dynamically during schedule generation) performs better than a preplanned routing. Rachamadugu et al. [14] have proposed a quantitative measure of sequence flexibility and have shown that perfect sequence flexibility improves system performance. Similar observations have been made by Lin and Solberg [15]. In most work to date, tools, pallets/fixtures and their availability are not modeled adequately. A static allocation of tools is usually assumed in these studies. However, some researchers have considered a limited tool magazine capacity and the changing of tools from a central tool storage [16]. Pallet and fixture limitations on the system performance have been examined by Ravi et al. [17].

One of the most important issues in the FMS scheduling problem is the type of scheme used to generate schedules. Sabuncuoglu and Hommertzheim [9] have identified two types of scheduling schemes: off-line and on-line. Off-line scheduling refers to scheduling all operations of available jobs for the entire planning horizon, whereas on-line scheduling attempts to schedule operations one at a time as needed. Because scheduling decisions are delayed until the last moment in the latter case, the term "real-time scheduling" is also used for on-line scheduling [18]. There are advantages and disadvantages associated with each approach. Some researchers argue that off-line scheduling produces better solutions than on-line methods due to its broader system view $[19,20]$.
However, scheduling can be a very tedious task with offline methods because of the difficulty of generating the schedule and updating it in a dynamic manufacturing environment [21]. In contrast, on-line methods require less computational effort. They can easily capture the important elements of the system as is discussed by $\mathrm{Mu}$ khopadhyay et al. [22]. However, they are myopic in nature and hence the quality of their solutions may not be as good as the off-line methods. One purpose of this study is to develop an off-line algorithm that can be used to investigate the research issues discussed above. This algorithm should not only consider the major elements of FMSs but also generate high quality schedules in a reasonable amount of time. In this paper, the basic structure and characteristics of such an algorithm is described and compared with on-line methods.

Most off-line scheduling algorithms proposed in the literature make a number of simplifying assumptions to reduce their computational burden. For example, some authors consider machines as a primary resource type $[5,6,23]$. In other studies, the emphasis is primarily on the aspect of scheduling the material handling in the FMS [24,25]. Nevertheless, there are also studies in which both machines and a material handling system are considered simultaneously in the scheduling process [26,27]. In all these studies, however, neither finite buffer capacity nor routing and sequence flexibilities are considered explicitly. Only routing flexibility has been exploited as in the work of Chang et al. [19], Hutchinson et al. [18] and De and Lee [28]. As compared to previously reported work, most of the scheduling factors (e.g., sequence and routing flexibilities) and resource types (e.g., machines and AGVs, buffers) are explicitly considered in our proposed algorithm. This enables us to model the real situation and compare the scheduling approaches (on-line and off-line) under more realistic environments.

Another important characteristic of the FMS scheduling problem is that buffer capacities at machines are limited. Hence, there is always a possibility of blocking in the system due to finite queue spaces. For example, Egbelu and Tanchoco [29] have discussed the conditions that lead to deadlock. Sabuncuoglu and Hommertzheim [30] and Leung and Sheen [31] have developed algorithms to resolve deadlocks in an FMS. The deadlock problem is also considered in this paper. Specifically, a new deadlock resolution mechanism is embedded in the proposed scheduling algorithm.

\section{The proposed algorithm}

The scheduling algorithm proposed in this paper is a heuristic based on the filtered beam search technique. Beam search is a fast and approximate branch and bound (B \& B) method which operates on a search tree. This partial enumeration technique uses heuristics to estimate 